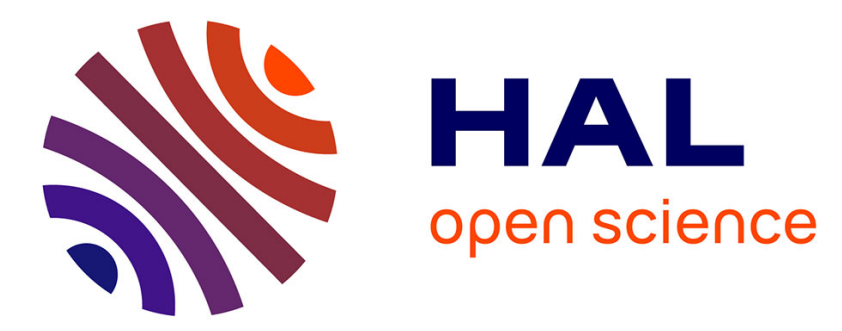

\title{
Effect of Industrial Impregnation Process on the Magnetic Properties of Electrical Steels
}

H. Helbling, Abdelkader Benabou, A. van Gorp, A. Tounzi, M. El Youssef, W. Boughanmi, D. Laloy

\section{- To cite this version:}

H. Helbling, Abdelkader Benabou, A. van Gorp, A. Tounzi, M. El Youssef, et al.. Effect of Industrial Impregnation Process on the Magnetic Properties of Electrical Steels. Journal of Magnetism and Magnetic Materials, inPress, 10.1016/j.jmmm.2021.167942 . hal-03182770

\section{HAL Id: hal-03182770 \\ https://hal.science/hal-03182770}

Submitted on 26 Mar 2021

HAL is a multi-disciplinary open access archive for the deposit and dissemination of scientific research documents, whether they are published or not. The documents may come from teaching and research institutions in France or abroad, or from public or private research centers.
L'archive ouverte pluridisciplinaire HAL, est destinée au dépôt et à la diffusion de documents scientifiques de niveau recherche, publiés ou non, émanant des établissements d'enseignement et de recherche français ou étrangers, des laboratoires publics ou privés. 


\title{
Effect of Industrial Impregnation Process on the Magnetic Properties of
}

\section{Electrical Steels}

H. Helbling ${ }^{1}$, A. Benabou ${ }^{1}$, A. Van Gorp ${ }^{2}$, A. Tounzi ${ }^{1}$, M. El Youssef ${ }^{2}$, W. Boughanmi ${ }^{3}$ and D. Laloy ${ }^{3}$

${ }^{1}$ Univ. Lille, Arts et Metiers Institute of Technology, Centrale Lille, Yncrea Hauts-de France, ULR 2697 - L2EP - Laboratoire d'Electrotechnique et d'Electronique de Puissance de Lille, Bât. ESPRIT, Avenue Henri Poincaré, 59655 Villeneuve d'Ascq, France.

${ }^{2}$ Arts et Métiers Institute of Technology, Mechanics, Surfaces and Materials Processing (MSMP), Lille, France.

${ }^{3}$ JEUMONT Electric, 59460 Jeumont, France

*Corresponding author e-mail: abdelkader.benabou@univ-lille.fr, Phone: +33(0)362268215

\begin{abstract}
This paper deals with the experimental investigation of the effect of impregnation process on the normal magnetization curve and iron losses of electrical steels. To address this issue, several laminated toroidal magnetic circuits have been designed to characterize the magnetic properties with the flux metric method. The first configuration considers magnetic circuits wrapped with adhesive tape so that the dielectric resin will be deposited only on the outer surface of the magnetic circuit. In the second configuration, the magnetic circuits are unwrapped, which will allow the resin to diffuse within the inter-laminar spaces of the magnetic circuit. The obtained experimental results show significant effects on the magnetic properties in both cases. However, depending on the considered configuration, the resin diffusion also has an influence on the changes in magnetic properties.
\end{abstract}

Keywords: Magnetic Properties; Iron Losses; Manufacturing Process; Impregnation Process

\section{Introduction}

The magnetic circuits of electrical machines play a significant role in the efficiency of the energy conversion process and also on the machine performances. Therefore, to be able to increase the energy efficiency of these machines, it is relevant to consider the magnetic core properties within the context of their operating conditions as well as their manufacturing processes. In this latter case, the impact of manufacturing processes on the magnetic 
properties of electrical steels may be significant. In the literature, the most commonly studied processes are the cutting, welding, pressing or shrink-fitting [1-5] of laminations or magnetic cores. However, for some specific industrial applications such as large alternators, the magnetic circuits are impregnated, under vacuum pressure followed by a heat treatment, with a dielectric resin. This impregnation process is realized once the magnetic circuits are built and wound. Its objectives are mainly to ensure the thermal properties, electrical insulation, mechanical withstand capability and also to reduce the vibrations of the electrical machine during operation [6], [7]. Preliminary considerations indicate that the resin will shrink around the magnetic circuit when cooled down after the heat treatment, thus requiring investigating its effect on the magnetic circuit properties. However, no study was conducted on the effect of this specific process on the magnetic properties.

In that context, the presented work investigates the effect of an industrial impregnation process on the normal magnetization curve and iron losses of electrical steel. Particularly, this paper makes a first link between the effects of an impregnation process on the magnetic properties and the diffusion of the resin within the magnetic core. To address this issue, several laminated toroidal magnetic circuits have been designed to characterize the magnetic properties before and after undergoing an industrial impregnation process. The magnetic measurements are performed with standard equipment based on the flux metric method.

This work presents several highlights. To our best knowledge, the proposed study constitutes the first investigation concerning the effect of impregnation process on the magnetic properties. The study shows a link between the level of resin diffusion within the magnetic circuit, the resin thermal contraction and the modifications of magnetic properties. Finally, the obtained experimental results, which have been consolidated by a repeatability analyzis, are analyzed and discussed relying in particular on thermo-mechanical simulations.

The paper is structured as follows. The second section describes the adopted methodology, including the presentation of the industrial impregnation process, the design of the studied samples, the magnetic measurement method and the experimental protocol that was followed. Then, results related to the effect of impregnation process on the iron losses and the normal magnetization curves are given. Moreover, thanks to thermo-mechanical finite element simulations, these effects are linked to the thermal retraction of the resin on the magnetic circuit. Finally, the main conclusions of the study and future work are presented.

\section{Methodology and experimental protocol}

\subsection{The studied industrial impregnation process}

Usually, large electrical machines, once the magnetic circuits are built and wound, are impregnated with a dielectric resin that is polymerized under heat treatment. As mentioned in the introduction, the aims of this process are mainly to ensure thermal properties, electrical insulation and reduction of the machine vibrations during operation. In this study, the considered industrial impregnation process involves two cycles as presented in Figure 1. Each cycle includes a vacuum pressure impregnation phase followed by a heat treatment phase. The impregnation phase consists in placing the machine in a tank which is subjected 
to a defined level of vacuum and applying the resin. The following heating phase consists in placing the impregnated machine in an oven under a temperature $T_{1}$ for duration $d_{1}$ (respectively $T_{2}$ and $d_{2}$ for the second cycle). During these operations, the conductive areas are protected by a coating.

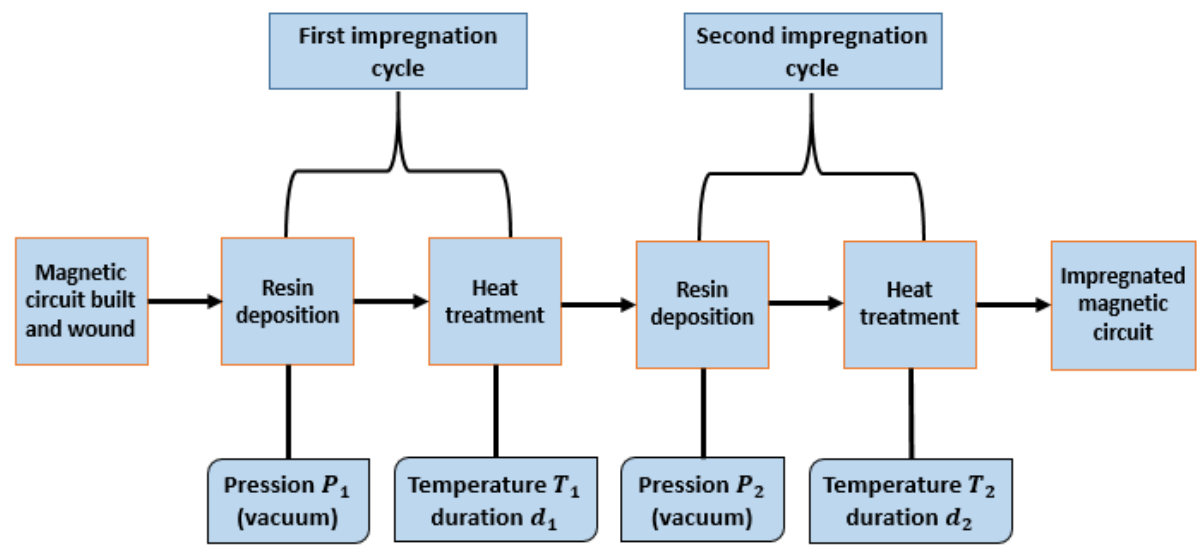

Figure 1 - Schematic diagram of the studied impregnation process

\subsection{Methodology}

The methodology of this work is as follows. First, four laminated toroidal magnetic circuits have been prepared for a characterization with the flux metric method. Two configurations are considered: the first one with two cores wrapped by a non-porous adhesive tape and the second one with two non-wrapped cores. In the first configuration, the nonporous adhesive tape will not allow the resin to diffuse within the magnetic circuits, which means that the resin is deposited only on the outer surface of the circuit. Without any tape, the resin will diffuse within the inter-laminar spaces of the magnetic circuit. All the magnetic cores are impregnated using the same process. Finally, the impregnated cores are characterized, including a repeatability analyzis concerning the impregnation process and the magnetic measurement method. A simplified schematic diagram of the methodology is presented in Figure 2.

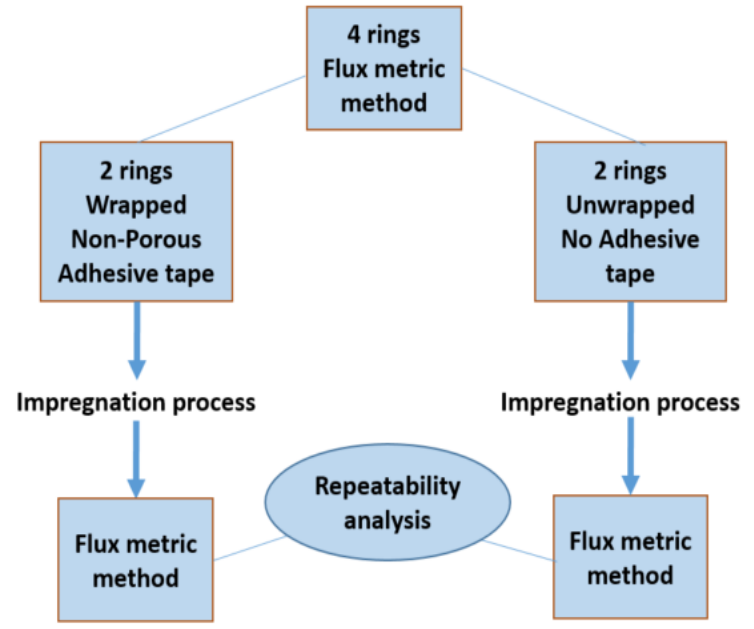

Figure 2 - Schematic diagram of the used methodology 


\subsection{Design of the magnetic cores}

All toroidal magnetic circuits come from the same coil of fully processed non-oriented electrical steel of grade $M 400-65 \mathrm{~A}$. This grade is commonly used for large electrical machines. Moreover, the toroidal magnetic circuits have been cut at the same time by wire electrical discharge machining (WEDM) to ensure a limited impact of the cutting method on the magnetic properties [8]. The inner and outer radii of the toroids are $86 \mathrm{~mm}$ and $100 \mathrm{~mm}$ respectively, and their height is $13 \mathrm{~mm}$ with 20 stacked strips of $0.65 \mathrm{~mm}$ thickness. The technical characteristics of the non-porous adhesive tape are given in Table 1. The maximum operation temperature of the non-porous adhesive tape is higher than the temperatures involved by the impregnation process. Regarding the dielectric resin, this latter is an epoxy based resin that is conventionally used for industrial impregnation processes.

As illustration, a ring core with the non-porous tape is shown after being impregnated in Figure 3.

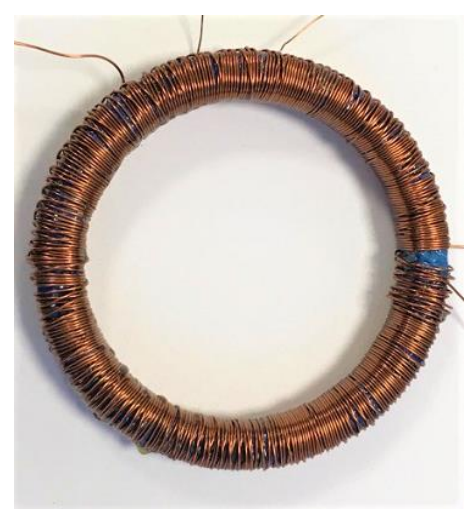

Figure 3 - Impregnated magnetic circuit with the non-porous adhesive tape

\begin{tabular}{|c|c|}
\hline Support type & Polyester \\
\hline Adhesive type & Silicone \\
\hline Total thickness (support + adhesive) & $63 \mu \mathrm{m}$ \\
\hline Maximum temperature & $204^{\circ} \mathrm{C}$ \\
\hline Elongation at break & $100 \%$ \\
\hline
\end{tabular}

Table 1 - Technical characteristics of the non-porous tape

\subsection{Magnetic Measurement}

Magnetic measurements are performed on the MPG200D equipment from Brockhaus in accordance with the standard IEC 60404-4 [9]. The repeatability of the measurements with the ring core tester equipment is less than $0.1 \%$.

We consider the configuration before the impregnation process as the reference case. The corresponding iron losses and normal magnetization curves are given in Figure 4 for all samples $\left(\mathrm{N}^{\circ} 1\right.$ to 4$)$. The iron losses under sinusoidal magnetic flux density of $1.5 \mathrm{~T}$ (peak) and $50 \mathrm{~Hz}$ are approximately $3.75 \mathrm{~W} / \mathrm{kg}$, which fulfils the requirements of the studied M400-65A electrical steel grade. It also means that the sample cutting did not affect significantly the material properties regarding the small width $(7 \mathrm{~mm})$ of the magnetic circuit. 

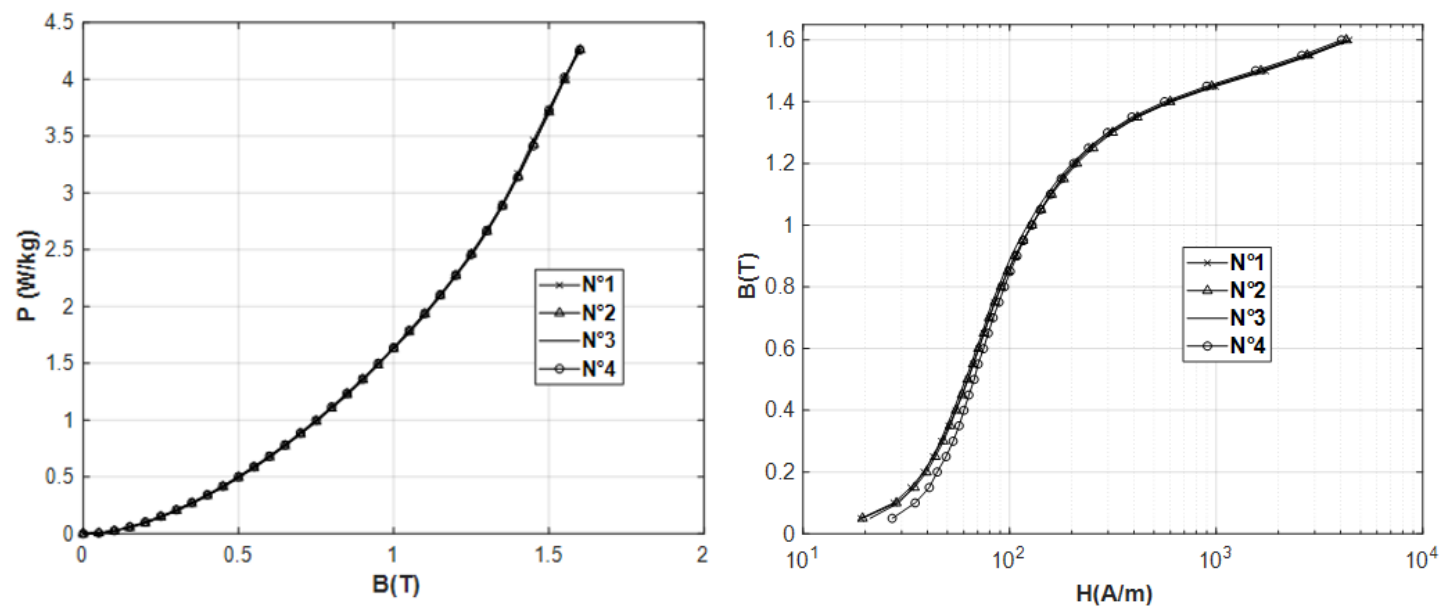

Figure 4 - Iron losses (left) and normal magnetization curves (right) at $50 \mathrm{~Hz}$ in the case of all toroidal samples before the impregnation process

\subsection{Experimental protocol}

The experimental protocol is as follows. First, the magnetic circuits are characterized before being impregnated. To reduce the risk of winding degradation and to avoid short circuits with the magnetic core, a first adhesive tape is wrapped on each ring core before putting the secondary winding. This latter is also wrapped by a second adhesive tape layer before placing the primary winding.

After this step, the circuits are separated into two groups: the first is composed of magnetic circuits which are unwound and unwrapped, leaving only the lamination stack. For the second group, neither the adhesive tape nor the winding are removed. The magnetic circuits of both groups are then impregnated. For the first group, the resin will be directly in contact with the magnetic cores and will diffuse within the inter-laminar spaces. Whereas, for the second group, due to use of a non-porous adhesive tape, the resin will remain on the outer surface of the magnetic cores.

The employed non-porous adhesive tape can withstand the temperatures $T_{1}$ and $T_{2}$ (see Figure 1) during the heat treatment phase of the impregnation process. Both groups undergo the full impregnation process and the magnetic cores of the first group are wound and wrapped again. Finally, the magnetic properties of all impregnated circuits are characterized to investigate the influence of the impregnation process. Measurements are carried out under sinusoidal magnetic flux density varying from $0.05 \mathrm{~T}$ to $1.6 \mathrm{~T}$ at given frequencies of $5 \mathrm{~Hz}, 50 \mathrm{~Hz}$ and $300 \mathrm{~Hz}$.

A first analyzis of the measurements shows that the error induced by the reproducibility of measurements is negligible compared to the effect of the impregnation process on the magnetic properties. In the most unfavorable case, the reproducibility is twenty times smaller than the variation observed following the impregnation process. Therefore, in the next section, and for more convenience, we will present the results for a single sample from each group.

\section{Results and discussion}




\subsection{Normal magnetization curves}

First, results concerning the normal magnetization curves are given at $50 \mathrm{~Hz}$ in Figure 5 and Figure 6. We observe that, with or without tape, the studied industrial impregnation process leads to the degradation of the normal magnetization curves. To emphasize this degradation, the relative increase of the magnetic field, to reach a given magnetic flux density, is calculated by considering the samples before the impregnation process as being the reference. For each induction level and each impregnation process, the variation $\Delta \mathrm{H}=\mathrm{H}_{1}-\mathrm{H}_{0}$, as illustrated in Figure 5, is determined; where $\mathrm{H}_{0}$ and $\mathrm{H}_{1}$ are the magnetic fields before and after impregnation respectively. The obtained relative differences $\Delta \mathrm{H} / \mathrm{H}_{0}$ are presented in Figure 7 for the frequencies $5 \mathrm{~Hz}, 50 \mathrm{~Hz}$ and $300 \mathrm{~Hz}$. These results show a degradation for low induction levels (linear part $<1 \mathrm{~T}$ ) and in the knee region (between 1T and 1.4T), with a more significant effect when the resin diffuses within the cores (without tape). However, the orders of magnitude of the degradation are relatively close between both configurations, especially in the knee region.

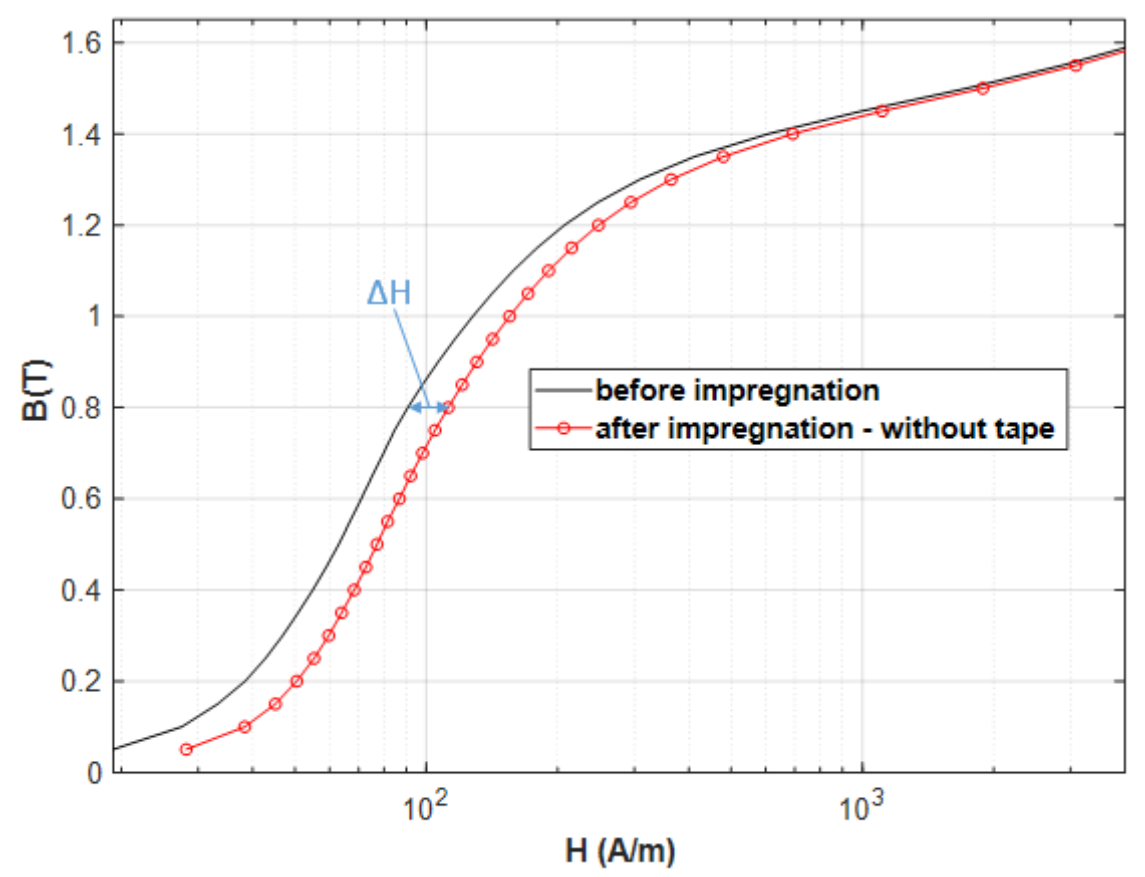

Figure 5 - Normal magnetization curves at $50 \mathrm{~Hz}$, before and after the impregnation process, for the magnetic circuit without tape (diffusion of the resin within the core) 


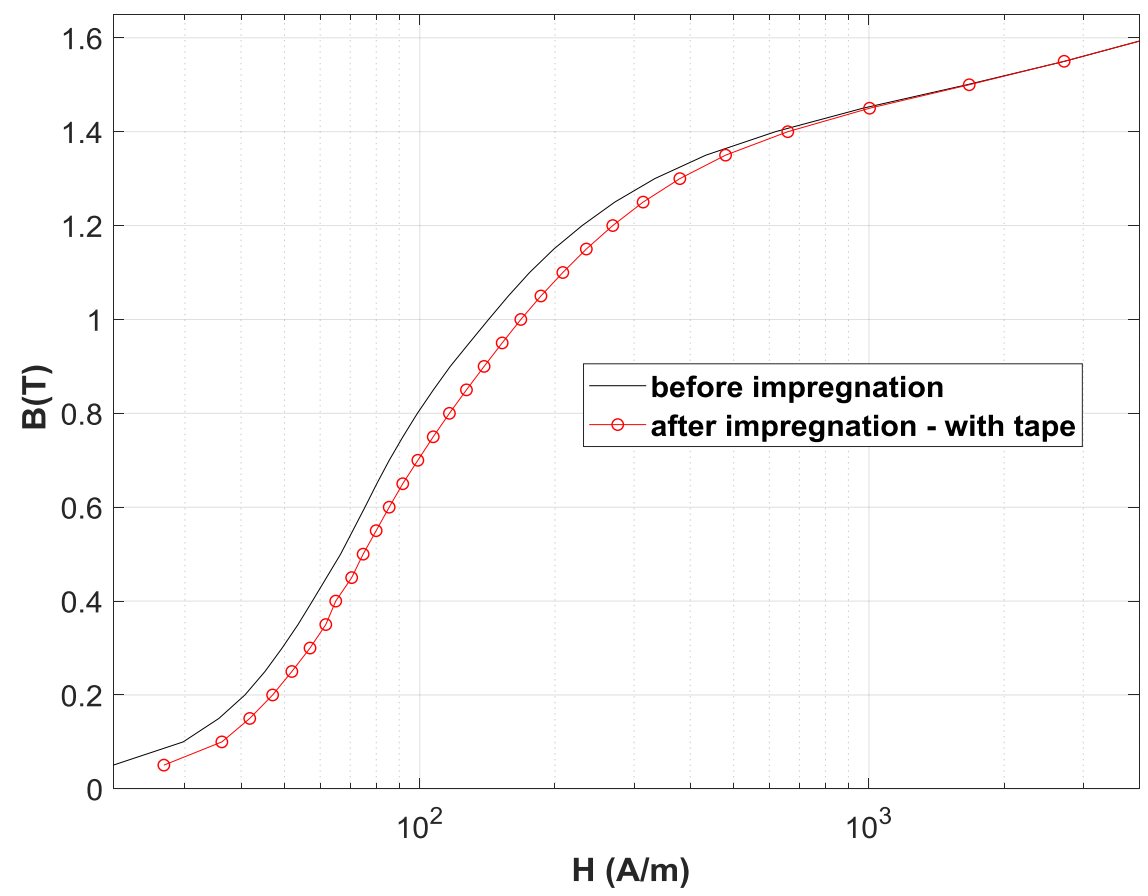

Figure 6 - Normal magnetization curves at $50 \mathrm{~Hz}$, before and after the impregnation process, for the magnetic circuit with tape (only surface diffusion of the resin)

We also observe in Figure 5 that the saturation levels of the normal magnetization curves before and after impregnation show a slight discrepancy whereas they are expected to be the same, regardless of the impregnation process. Indeed, the saturation depends mainly on the composition of the magnetic material, which has not been modified in the process. The observed discrepancy in the saturation region of Figure 5 can be explained by a more significant impact of the impregnation process even in the high excitation field region. To address this issue, magnetic measurements were carried out to $1.75 \mathrm{~T}(\mathrm{H} \approx 12000 \mathrm{~A} / \mathrm{m})$ and reported in Figure 7 . The relative difference $\Delta \mathrm{H} / \mathrm{H}_{0}$ tends to zero, meaning that the saturation level is indeed the same, even for the case without tape. In addition to previous observations, in Figure 8, the relative difference on the magnetic field is summarized for frequencies $5 \mathrm{~Hz}$, $50 \mathrm{~Hz}$ and $300 \mathrm{~Hz}$. It appears that the level of degradation decreases when the frequency increases. This observation can be explained by the required magnetic field $\mathrm{H}=\mathrm{H}_{\text {hyst }}+\mathrm{H}_{\text {dyn }}$ to magnetize the material in the dynamic regime. Indeed, with increasing frequency and to reach a given induction level, the dynamic field $\mathrm{H}_{\mathrm{dyn}}$ becomes more predominant with regard to the static field $\mathrm{H}_{\text {hyst }}$. This suggests that the static field contribution is the one mostly affected by the impregnation process, a result that is corroborated with the loss behavior detailed in next section.

Previous results show clearly that the impregnation process degrades the magnetic properties on the whole range of the magnetization process of the material. Also, these observations indicate that the way the resin diffuses, or not, within the magnetic core, has a more or less significant influence on the magnetic properties. Indeed, as each core was subjected to the same impregnation process, the only difference is the ability of the resin to diffuse either only on the outer surface or within the magnetic circuit. 


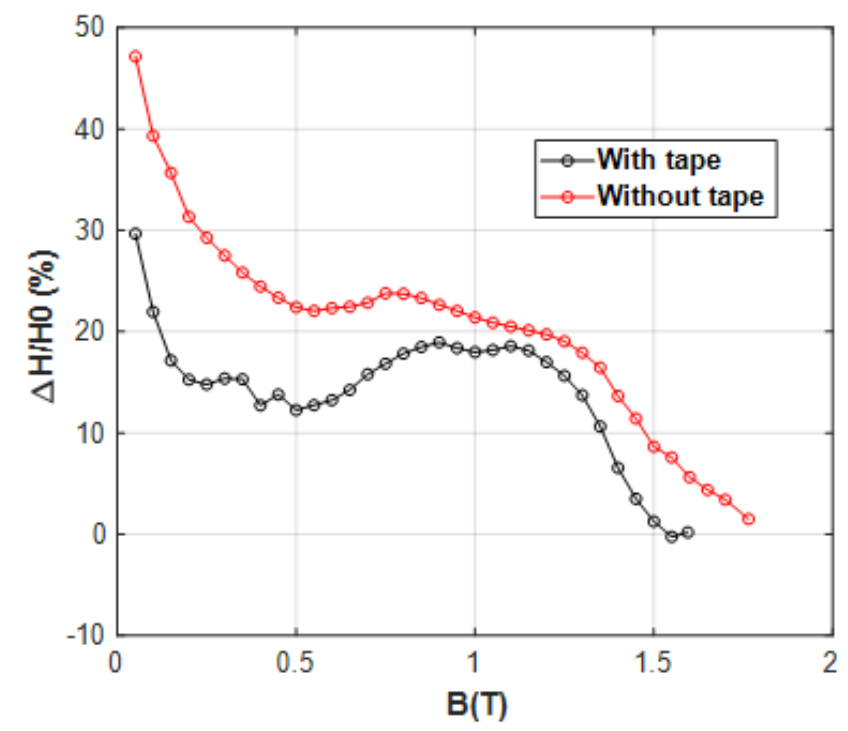

Figure 7 - Relative difference on magnetic field, due to the impregnation process with and without adhesive tape, at $50 \mathrm{~Hz}$
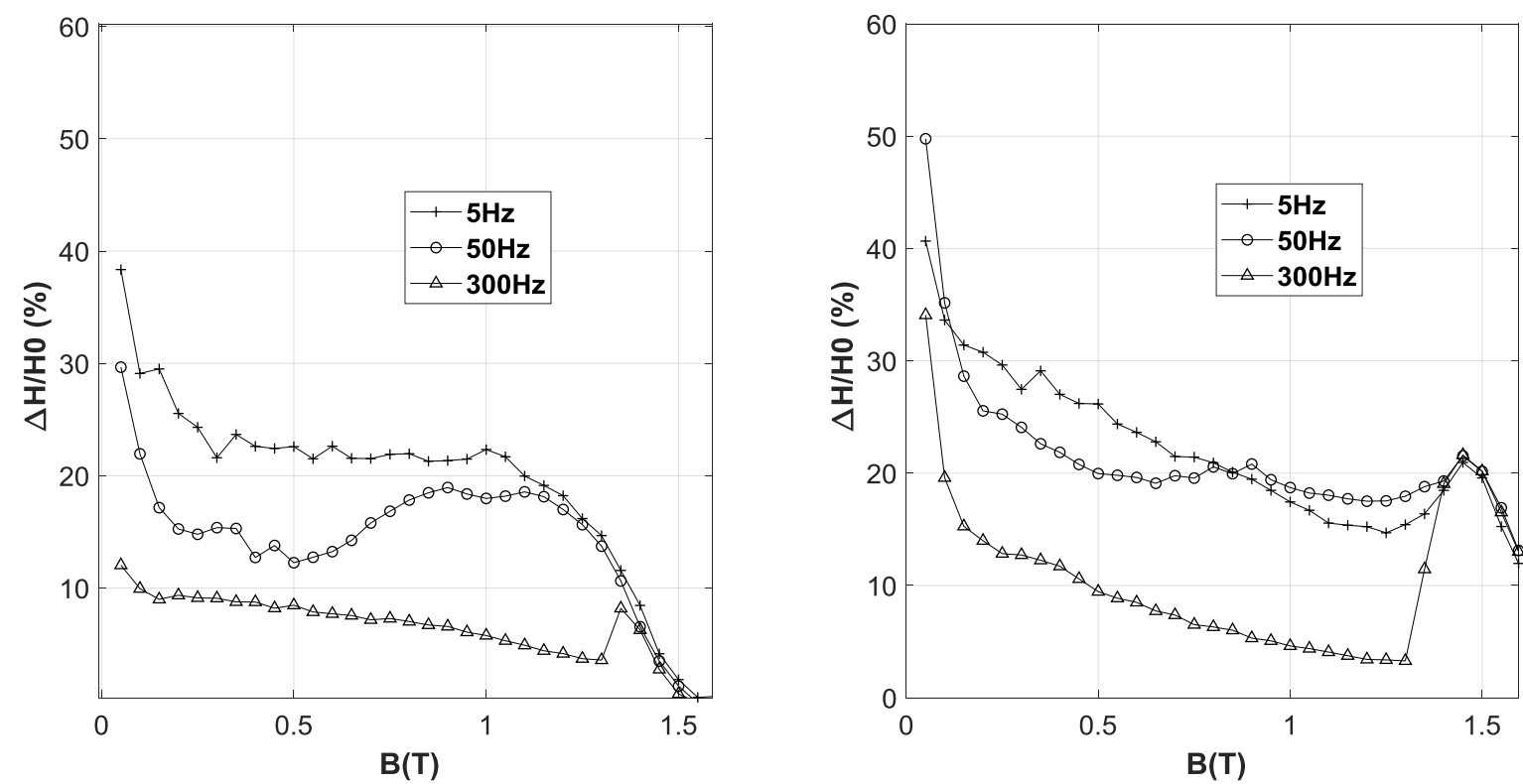

Figure 8 - Relative difference on the magnetic field due to the impregnation process with (left) and without (right) adhesive tape

Finally, to summarize the observed effects of impregnation, experimental values of the required magnetic field are given in Table 2 for the induction levels $0.5,1$ and $1.5 \mathrm{~T}$

\begin{tabular}{|c|c|c|c|}
\hline & $0.5 \mathrm{~T}$ & $1 \mathrm{~T}$ & $1.5 \mathrm{~T}$ \\
\hline \multicolumn{4}{|c|}{ Sample with non-porous tape } \\
\hline Magnetic field before Impregnation Process (A/m) & 67 & 142 & 1650 \\
\hline Magnetic field after Impregnation Process (A/m) & 75 & 168 & 1659 \\
\hline \multicolumn{4}{|l|}{ Sample without tape } \\
\hline Magnetic field before Impregnation Process $(\mathrm{A} / \mathrm{m})$ & 65 & 142 & 1650 \\
\hline Magnetic field after Impregnation Process (A/m) & 80 & 172 & 1790 \\
\hline
\end{tabular}

Table 2 - Experimental values, before and after impregnation process, of the required magnetic field to reach induction levels of $0.5 \mathrm{~T}, 1 \mathrm{~T}$ and $1.5 \mathrm{~T}$ 


\subsection{Iron losses}

Another sensitive indicator of the process influence on the magnetic properties is given by the iron losses. The impact of the impregnation process on these losses is shown in Figure 9 and Figure 10 for both considered configurations. As for the normal magnetization curve, the effect on power losses is given by the ratio $\Delta \mathrm{P} / \mathrm{P}_{0}$, where $\mathrm{P}_{0}$ represents the losses before the impregnation process and $\Delta \mathrm{P}$ represents the difference on power losses, before and after the impregnation process. These results show that the impregnation process leads to a significant increase of the iron losses.

In Figure 10, a zoom of Figure 9 is given for induction levels above 0.4T, which are more relevant magnetization regions for energy conversion devices. The increase of iron losses is about two times greater when the resin diffuses within the magnetic circuit, compared to the case where the resin is deposited only on the surface. This result shows that the impact on the magnetic properties depends on the way in which the resin diffuses within the magnetic circuit.

Moreover, the lower the frequency is, the more the total iron losses increase. This observation strongly suggests that, among the three loss contributions (hysteresis, classical and excess losses [10]), the hysteresis loss contribution is the one mostly affected by the impregnation process. In order to confirm this assumption, the evolution of the hysteresis losses must be extracted from the measurements. Therefore, to determine the hysteresis loss contribution, several measurements were made at the following frequencies: 5, 20, 50, 150, 200 and $300 \mathrm{~Hz}$. Then, the specific energy (total power losses divided by the frequency) is extrapolated to zero frequency for each level of induction in order to determine the static (hysteresis) contribution of the losses. Finally, and similarly to previous analyzes, the relative difference of the hysteresis losses $\Delta \mathrm{P}_{\mathrm{Hyst}} / \mathrm{P}_{\mathrm{Hyst} 0}$ is calculated and given in Figure 11 .

According to Figure 10 and Figure 11, it appears that the effect of the impregnation process on the hysteresis losses is significant and very close to that observed on the total iron losses. These results confirm that the hysteresis losses are the most impacted by the impregnation process. 

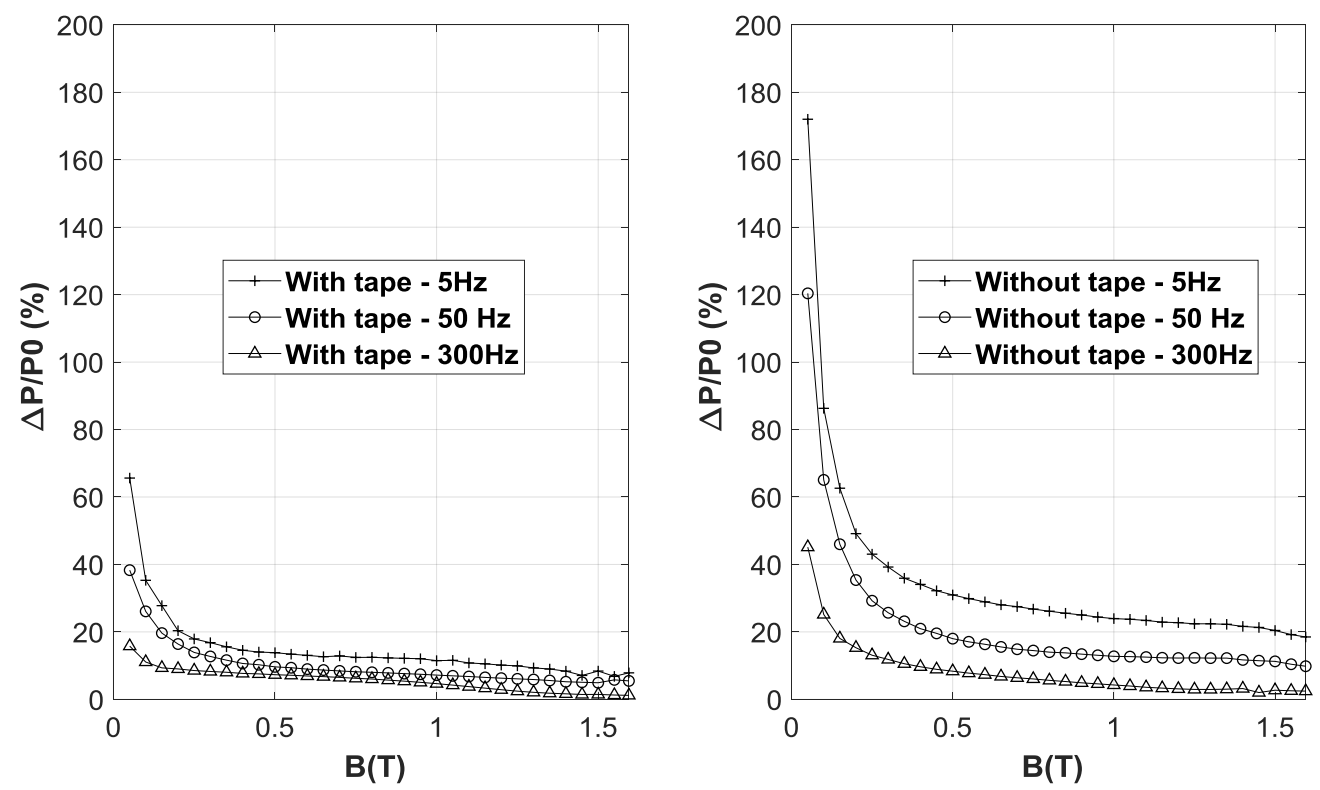

Figure 9 - Relative difference on iron losses, due to impregnation process with (left) and without (right) tape, for $5 \mathrm{~Hz}, 50 \mathrm{~Hz}$ and $300 \mathrm{~Hz}$
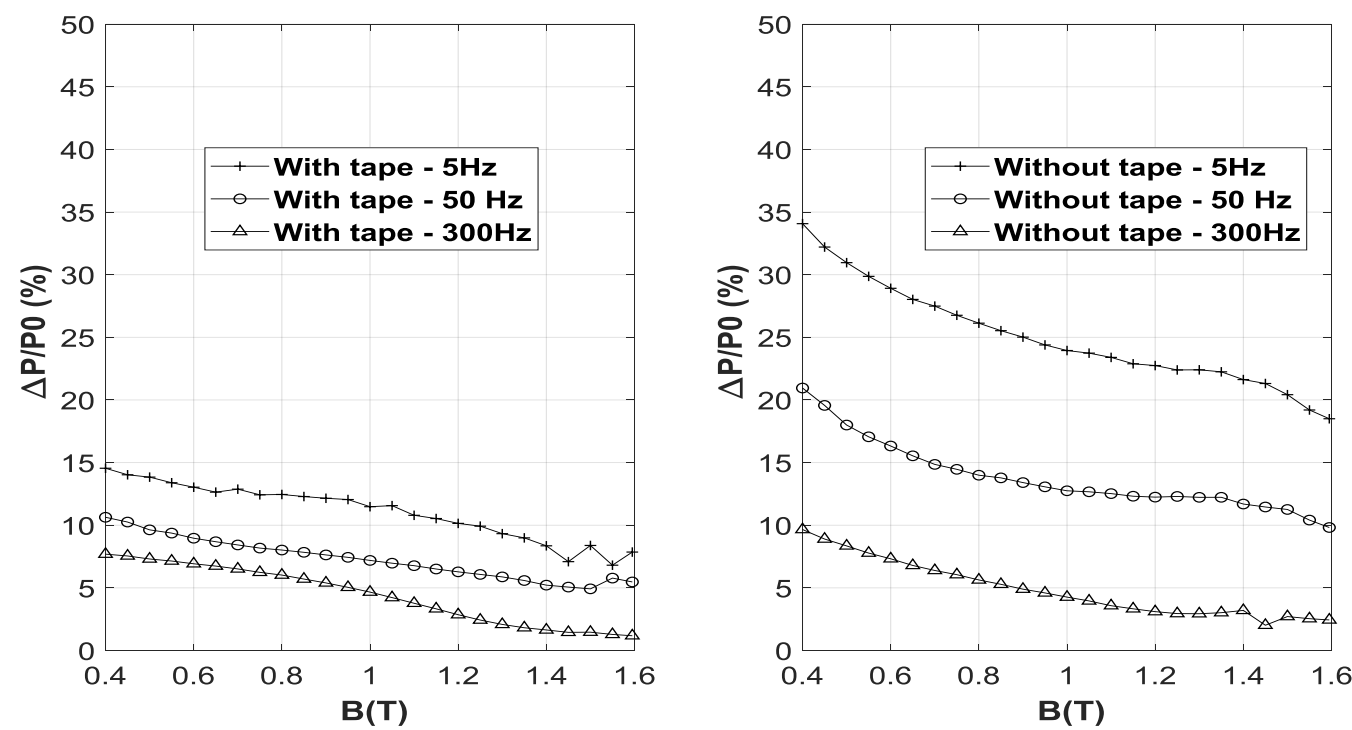

Figure 10 - Relative difference on iron losses, due to impregnation process with (left) and without (right) tape, for average and high induction levels and for $5 \mathrm{~Hz}, 50 \mathrm{~Hz}$ and $300 \mathrm{~Hz}$ 

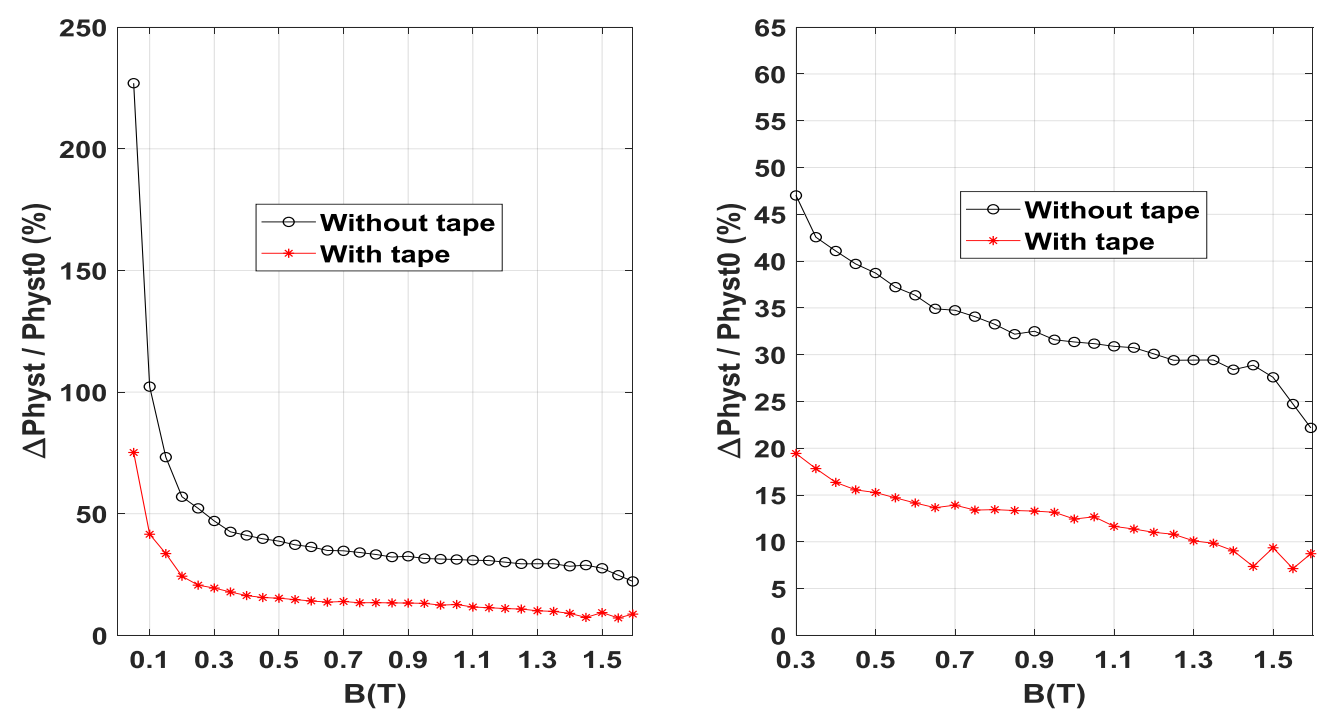

Figure 11 - Relative difference on hysteresis losses, due to impregnation process with and without tape, for all induction levels (left) and for average and high induction levels (right)

Finally, we give in Table 3 the power losses values for $0.5,1$ and $1.5 \mathrm{~T}$ before and after the impregnation process.

\begin{tabular}{|c|c|c|c|}
\hline & $0.5 \mathrm{~T}$ & $1 \mathrm{~T}$ & $1.5 \mathrm{~T}$ \\
\hline \multicolumn{4}{|c|}{ Sample with non-porous tape } \\
\hline Iron losses before Impregnation Process (W/kg) & 0.52 & 1.7 & 3.86 \\
\hline Iron losses after Impregnation Process (W/kg) & 0.57 & 1.83 & 4.05 \\
\hline \multicolumn{4}{|l|}{ Sample without tape } \\
\hline Iron losses before Impregnation Process (W/kg) & 0.51 & 1.7 & 3.85 \\
\hline Iron losses after Impregnation Process (W/kg) & 0.61 & 1.95 & 4.35 \\
\hline
\end{tabular}

Table 3- Experimental values of the iron losses before and after impregnation process for induction levels of $0.5 \mathrm{~T}, 1 \mathrm{~T}$ and $1.5 \mathrm{~T}$

\subsection{Discussion}

To further analyze all previous results, one has to first consider the resin mechanical properties. According to the literature, the coefficient of thermal expansion of an epoxy resin is about four times greater than that of steel. After the heat treatment of the impregnation process, the magnetic circuit and the resin will cool down at the same time, which means that the resin will shrink much more than the steel because of its higher coefficient of thermal expansion. Thus, a mechanical stress will be induced on the magnetic circuit. To estimate this mechanical stress, a thermo-mechanical Finite Element (FE) study is performed with the software Abaqus [11]. The case of study corresponds to a magnetic circuit (having the same size as those used for the experimental measurements) surrounded by one millimeter of resin thickness. The FE simulation is a coupled temperature-displacement problem, with an initial temperature equivalent to $T_{2}$ and a final temperature corresponding to $23^{\circ} \mathrm{C}$. The mechanical and thermal characteristics used for the FE simulations are given in Table 4. The studied geometry has an axial symmetry around $(\mathrm{Oz})$ (see Figure 12 ), thus we have considered one 
fourth of the device with the planes $(x O z)$ and $(y O z)$ as boundary conditions. Also, the studied device has a plane of symmetry parallel to $(x O y)$ that allows representing only half of the device along the height. A zero displacement condition is imposed on this symmetry plane.

\begin{tabular}{|c|c|c|c|}
\hline & Unit & Magnetic circuit & Resin \\
\hline Young's modulus & $\mathrm{GPa}$ & 211 & 5.9 \\
\hline Poisson's ration & - & 0.29 & 0.35 \\
\hline Mass Density & $\mathrm{kg} \cdot \mathrm{m}^{-3}$ & 8050 & 1250 \\
\hline Thermal conductivity & $\mathrm{W} \cdot \mathrm{m}^{-1} \cdot \mathrm{K}^{-1}$ & 50,2 & 0.25 \\
\hline Temperature expansion coefficient & $\mathrm{K}^{-1}$ & $1.10^{-5}$ & $4.10^{-5}$ \\
\hline Specific Heat & $\mathrm{J} . \mathrm{kg}^{-1} \cdot{ }^{\circ} \mathrm{K}^{-1}$ & 420 & 1000 \\
\hline
\end{tabular}

Table 4 - Mechanical and thermal characteristics of the FE simulation
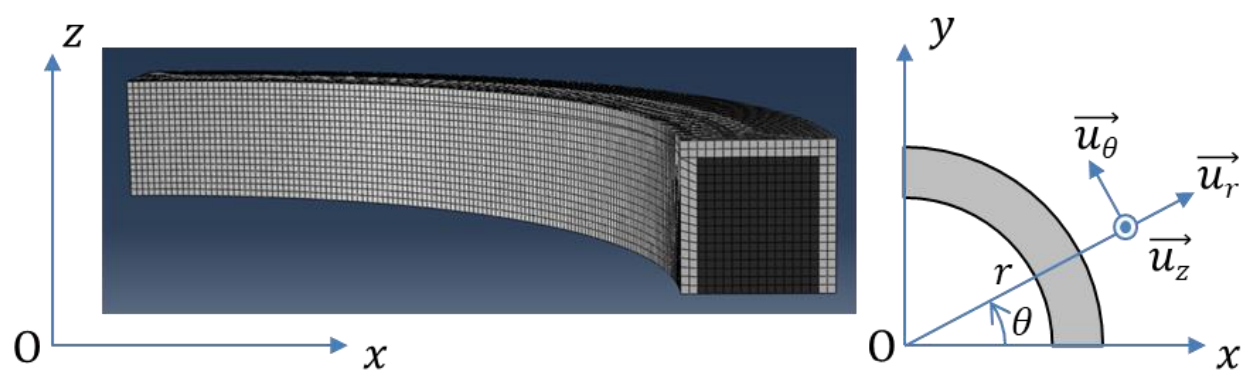

Figure 12 - View of the magnetic circuit (black) and the resin (grey) - Perspective view (left) and top view (right)

The results in Figure 13 show the mechanical stresses along the magnetic flux direction, denoted $\sigma_{\theta}$ (Figure 13-a), orthogonal to the flux direction, denoted $\sigma_{r}$ (Figure 13b), and along the height of the magnetic circuit, denoted $\sigma_{z}$ (Figure 13-c). In Figure 13-a, we observe that the stress along the flux direction is quite homogeneous within the magnetic circuit with a compressive stress around $-15 \mathrm{MPa}$. Still, Figure 13-b shows that in the plane of the laminations the stress, in the direction orthogonal to the flux direction, is near zero at the center of the magnetic core and progressively increases to a compressive value of $-15 \mathrm{MPa}$ (light blue) or -30MPa (dark blue). Along the height direction (Figure 13-c), the compressive stress is relatively homogeneous and its value is about -10MPa. Thus, considering the three directions, the magnetic core is exclusively subjected to compressive stress. 


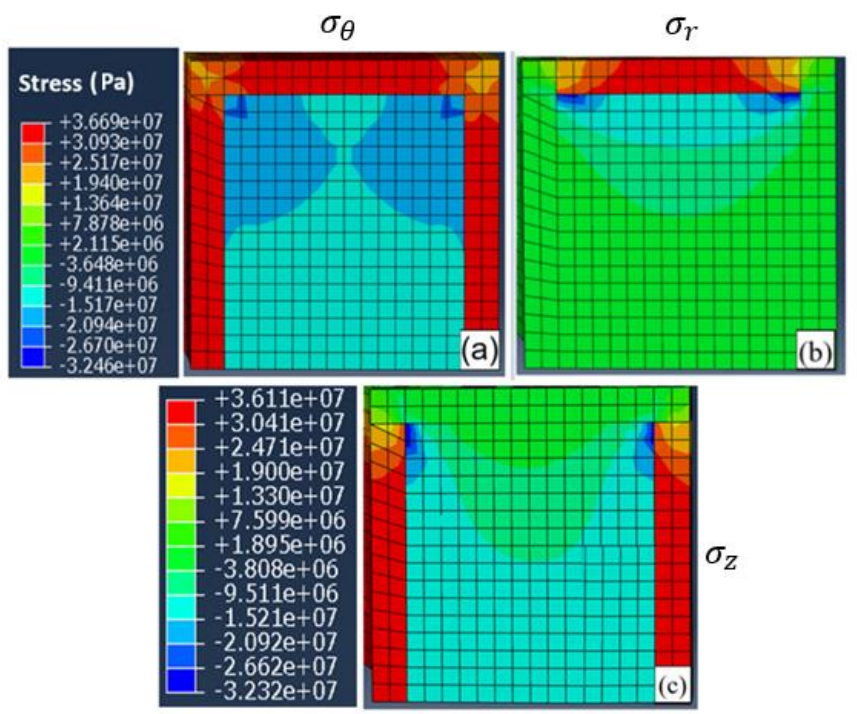

Figure 13 - Induced stress in the cross section due to the thermal contraction of the resin flux direction (a), orthogonal to the flux direction (b) and along the height of the lamination stack (c)

It is well established in the literature [12-15] that mechanical stress influences the magnetic behavior. Indeed, when considering the magnetic field in the same direction as the applied stress, a tensile stress can lead to slight magnetic improvements for low stress levels before deteriorating the magnetic behavior above a threshold. Regarding the compressive stress, this one leads to a continuous degradation effect that is, at the same stress level, more pronounced than the effect of the tensile stress [12-14]. In the present study, the compressive stress levels, as suggested by the thermo-mechanical FE simulations, are indeed a source of degradation for the magnetic properties, as also observed in the literature for compressive stresses applied in the lamination plane [15].

One must also note that the study has been carried out on ring-shaped magnetic cores whereas the effect of impregnation process is expected to be strongly related to the geometry of the magnetic circuit. Indeed, the way the resin will diffuse, and consequently its impact on the magnetic properties, is directly linked to the geometry of the core.

\section{Conclusions}

The presented study deals with a first investigation of the effect of an industrial impregnation process on the magnetic properties of a lamination stack. In particular, the changes in magnetic properties have been related to the way the resin diffuses within the magnetic circuit. For this, two groups of magnetic cores have been studied: a first group with ring cores that are wrapped by a non-porous adhesive tape, implying that the resin will only be distributed on the outer surface and a second group without tape, implying that the resin will diffuse within the magnetic core. Experimental results show that in both cases the impregnation process has a significant impact on the magnetic properties. In particular, they show that the more the resin diffuses within the magnetic circuit, the more the magnetic properties are deteriorated. Finally, the results of this study demonstrate the interest of 
investigating the impact of an impregnation process on magnetic properties of laminated magnetic cores.

One must note that the level of impact in the presented results is valid for the studied geometries, namely magnetic circuits of small sizes. Indeed, for larger magnetic circuits, in particular in the case of electrical machines, it is necessary to investigate whether and how the resin will diffuse over the entire magnetic circuit. However, this first investigation that shows the effect of the impregnation process on magnetic properties has many perspectives. In particular, the effect of impregnation can be accounted for in magnetic models of materials (loss model and normal magnetization curve). These models are required to further analyze the effect of impregnation process on electrical machine performances. Finally, studies need to be carried out in order to investigate to what extent the resin diffuses within the magnetic circuit, especially by investigating the diffusion process between the laminations. This way, the effect of resin diffusion process in magnetic cores with larger dimensions, such as those of high power electrical machines, can be accurately predicted.

\section{References}

[1] M. Bali, A. Muetze, Modeling the effect of cutting on the magnetic properties of electrical steel sheets, IEEE Trans. Ind. Electron. 64 (3) (2017) 2547-2556.

[2] K. Bourchas and al., Influence of cutting and welding on magnetic properties of electrical steels, 2016 XXII International Conference on Electrical Machines (ICEM), 2016, pp. 1815-1821.

[3] A. Schoppa, J. Schneider, C.-D. Wuppermann, T. Bakon, Influence of welding and sticking of laminations on the magnetic properties of non-oriented electrical steels, J. Magn. Magn. Mater. 254-255 (2003)

[4] K. Yamazaki and W. Fukushima, Loss analysis of induction motors by considering shrink fitting of stator housings, IEEE Trans. Magn. 51 (3) (2015) 1-4.

[5] H. Helbling, A. Benabou, A. Van Gorp, M. El Youssef, A. Tounzi, W. Boughanmi and D. Laloy, Effect on magnetic properties of inhomogeneous compressive stress in thickness direction of an electrical steel stack, J. Magn. Magn. Mater. 500 (2020) 166353

[6] S. Ayat, R. Wrobel, J. Goss and D. Drury, Estimation of Equivalent Thermal Conductivity for Impregnated Electrical Windings Formed from Profiled Rectangular Conductors, PEMD 2016.

[7] R. K. Singal, K. Williams and S. P. Verma, The effect of windings, frame and impregnation upon the resonant frequencies and vibrational behavior of an electrical machine stator, Experimental Mechanics, Vol. 30, p. 270-280, 1990,

[8] V. Manescu Paltanea, G. Paltanea and H. Gavrila, Energy loss analysis and magnetic properties of non-oriented electrical steel cut through different technologies, Adv. Mater. Res. (2015).

[9] International Standard IEC 60404-4. Magnetic Materials - Part 4: Methods of Measurements of d.c. Magnetic Properties of Iron Steel, IEC Stand. Publ., 2008.

[10] G. Bertotti, General properties of power losses in soft ferromagnetic material, IEEE Trans. on Mag., 24 (1) (1988), 621-630.

[11] ABAQUS, ABAQUS Documentation, Dassault Systemes, Providence, RI, USA, 2011.

[12] G.H. Shirkoohi, A.J. Moses, Effects of stress on magnetostrictive properties of low silicon non-oriented electrical steel, J. Magn. Magn. Mater. 83 (1) (1990) 177-178. 
[13] A.P.S. Baghel and al., An alternative approach to model mechanical stress effects on magnetic hysteresis in electrical steels using complex permeability, Comput. Mater. Sci. 166 (2019) 96-104.

[14] D. Miyagi, K. Miki, M. Nakano, N. Takahashi, Influence of compressive stress on magnetic properties of laminated electrical steel sheets, IEEE Trans. Magn. 46 (2) (2010) 318-321.

[15] U. Aydin, P. Rasilo, F. Martin, A. Belahcen, L. Daniel, A. Haavisto, A. Arkkio, Effect of multi-axial stress on iron losses of electrical steel sheets, J. Magn. Magn. Mater. 469 (2019) 